${ }^{1}$ Centro de Nutrición Molecular y Enfermedades Crónicas, Escuela de Medicina, Pontificia Universidad Católica de Chile. Santiago, Chile.

2Departamento de Nutrición, Diabetes y Metabolismo, Escuela de Medicina, Pontificia Universidad Católica de Chile. Santiago, Chile.

Recibido el 9 de noviembre de 2015, aceptado el 25 enero de 2016.

Correspondencia a: Dr. Attilio Rigotti Departamento de Nutrición, Diabetes y Metabolismo, Escuela de Medicina, Pontificia Universidad Católica, Marcoleta 367, interior, 2do Piso, Santiago, Chile.

Código Postal 8330024, Chile. Teléfono +56223543832 arigotti@med.puc.cl

\section{Evidencia actual sobre los beneficios de la dieta mediterránea en salud}

\author{
CATALINA DUSSAILLANT ${ }^{1}$, GUADALUPE ECHEVERRÍA ${ }^{1}$, \\ INÉS URQUIAGA ${ }^{1}$, NICOLÁS VELASCO ${ }^{2}$, ATTILIO RIGOTTI ${ }^{1,2}$ \section{of the mediterranean diet} \\ Current evidence on health benefits
}

The Mediterranean diet is currently considered a functional diet with an increasing amount of scientific evidence that supports its beneficial effects in human health. Several observational cross-sectional and prospective cohort studies show an association between this diet and a lower prevalence and incidence of chronic diseases, such as cardiovascular disease, cancer, metabolic syndrome, diabetes, and neurodegenerative diseases as well as a reduced overall mortality. Additionally, clinical interventional studies, particularly the PREDIMED (Prevención con Dieta Mediterránea) initiative, have shown, with high quality scientific evidence, that a Mediterranean diet-supplemented either with olive oil or nuts- can lower by 30\% the incidence of cardiovascular disease, reverse the metabolic syndrome, and prevent the development of diabetes and aging-related cognitive decline. Chile has one of the five Mediterranean ecosystems in the world, and therefore the implementation of this food pattern and lifestyle in our country may determine large benefits to the health status and quality of life in the Chilean population.

(Rev Med Chile 2016; 144: 1044-1052)

Key words: Diet, Mediterranean; Evidence-Based Medicine; Health Impact Assessment; Life Style.
$\Lambda$ ctualmente el estilo de vida se ha transformado en un factor fundamental en prevención y tratamiento de patologías crónicas relacionadas con riesgo cardiovascular, como la diabetes y el síndrome metabólico. Los factores dietéticos pueden modificar el perfil de riesgo poblacional para enfermedades crónicas y la hipótesis que asocia una dieta adecuada con salud cardiovascular ha ganado cada vez mayor aceptación en el mundo científico ${ }^{1}$.

La dieta mediterránea (DMed) es considerada uno de los patrones dietarios con mayor evidencia científica acumulada en cuanto a sus beneficios en salud humana, siendo cada vez mayor el interés del mundo científico en el estudio de su rol preventivo y como tratamiento en diversas patologías asociadas a inflamación crónica, como síndrome metabólico (SM), diabetes, enfermedad cardiovascular (ECV), enfermedades neurodegenerativas y cáncer, entre otras ${ }^{2}$. De hecho, en las últimas décadas, el número de publicaciones en el tema ha crecido en forma exponencial, alcanzando cerca de 500 artículos en Pubmed en el año 2014 (Figura 1).

Para medir la adherencia de una población a este patrón dietario, se han desarrollado y validado varios índices, entre ellos la Escala de Dieta Mediterránea ${ }^{3}$, el Î́ndice de Adecuación Mediterráneo ${ }^{4}$ y el Índice $S U^{5}$, que permiten asociar adherencia a esta dieta con diferentes eventos clínicos y objetivos en salud. Estos incluyen los componentes claves de la dieta mediterránea (pescado, cereales integrales, frutas, verduras, frutos secos, aceite de oliva y vino entre otros). Incluso, el índice desarrollado por Trichopulou y cols. fue adaptado y 


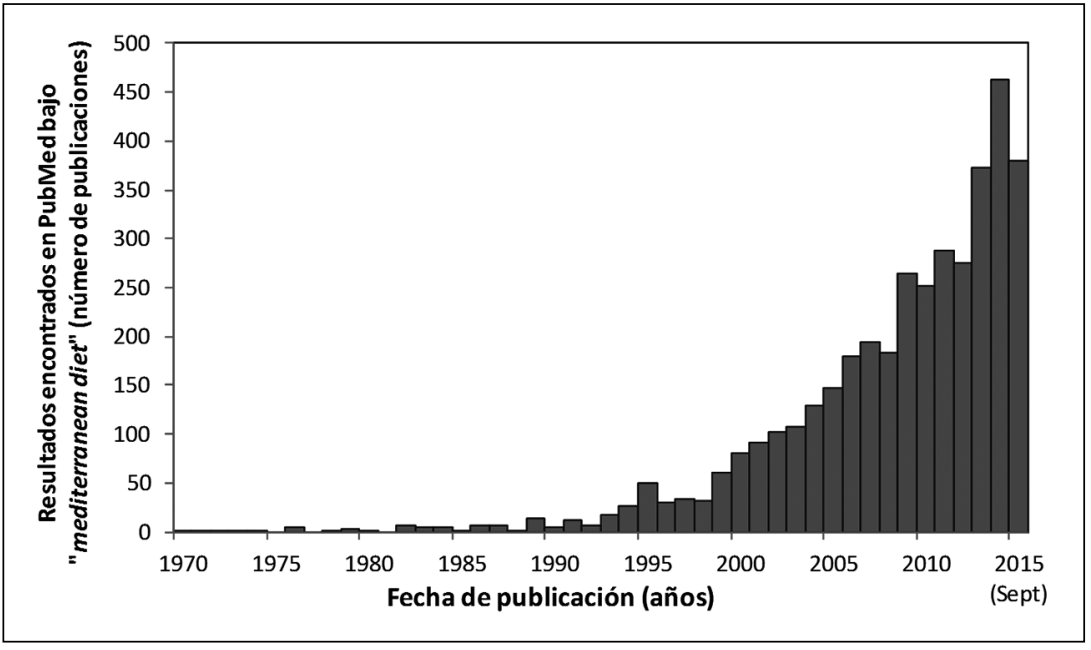

Figura 1. Evolución temporal del número de publicaciones sobre dieta mediterránea reportadas en PubMed desde 1970. aplicado en población estadounidense ${ }^{6}$. Utilizando estos índices, numerosos estudios transversales y de cohortes han asociado este patrón alimentario con menor incidencia de ECV, SM, cáncer y enfermedades neurodegenerativas así como menor mortalidad total y/o por estas patologías ${ }^{3,7-10}$.

\section{Evidencia acumulada de estudios transversales y cohortes prospectivas}

\section{Dieta mediterránea, enfermedad cardiovascular,} diabetes y síndrome metabólico

La cohorte griega del estudio EPIC (European Prospective Investigation into Cancer and Nutrition Study) es una de las observaciones de mayor impacto en cuanto a la relación entre el consumo de una DMed y ECV. En este estudio, un aumento de 2 puntos en el índice de adherencia a esta dieta, se asoció a una reducción de 33\% en mortalidad por $\mathrm{ECV}^{11}$. Adicionalmente, el análisis de una subcohorte de 2.700 individuos mayores de 60 años con antecedente de infarto al miocardio demostró que una mayor adherencia a la DMed se traducía en una reducción de $18 \%$ en la mortalidad total ${ }^{12}$. Otros estudios han confirmado estas asociaciones, entre ellos el seguimiento de una cohorte española de 13.600 adultos sin enfermedad coronaria. Después de 5 años, se observó que 2 puntos de incremento en adherencia a la DMed se asociaban a una disminución de $26 \%$ en el riesgo coronario ${ }^{13}$. Asimismo, la subcohorte española del estudio EPIC observó que aquellos individuos con mayor adherencia a esta dieta tenían menor incidencia de eventos coronarios $(\mathrm{RR}=0,60)$ comparado con los que tenían poca adherencia ${ }^{14}$. El efecto protector de la DMed en ECV ha sido observado también en poblaciones no mediterráneas, como el estudio de Mitrou y cols. que demostró que una mayor adherencia a la DMed se asociaba a una menor mortalidad por ECV $(\mathrm{RR}=0,78)$ durante el seguimiento por 10 años de 380.000 hombres y mujeres de Estados Unidos de Norteamérica ${ }^{15}$.

Asimismo, y confirmando los hallazgos previos, dos meta-análisis publicados recientemente -con los estudios prospectivos de grandes cohortes basados en eventos clínicos (incidencia o mortalidad) - indican que el incremento de dos puntos en una escala (rango de 0 a 18 puntos) de adherencia a la DMed se correlaciona con una reducción significativa de $10 \%$ en la incidencia o mortalidad cardiovascular ${ }^{10,16}$.

Por otro lado, el SM es un conjunto de factores de riego que aumentan el riesgo de enfermedad cardiovascular y diabetes. Un meta-análisis -con datos de 50 estudios y un total de 535.000 individuos- concluyó que la adherencia a DMed se asocia a $31 \%$ menor riesgo de SM, con un beneficio sobre todos sus componentes (hiperglicemia, obesidad abdominal, hipertensión arterial y dislipidemia aterogénica $)^{17}$. Otro meta-análisis de 2.650 individuos ha reportado que la DMed es más efectiva -que una dieta baja en grasas- en mejorar diferentes factores de riesgo cardiovascular así como varios parámetros inflamatorios ${ }^{18}$. 
Con respecto a diabetes, una mayor adherencia a la DMed se asocia a una menor incidencia de esta enfermedad así como a un mejor control metabólico y mortalidad total en este grupo de pacientes ${ }^{19-21}$. Por ejemplo, el seguimiento de la cohorte SUN (Seguimiento Universidad de Navarra) en 13.380 adultos estableció una relación inversa entre consumo de una DMed e incidencia de diabetes, con una disminución del riesgo de 35\% por cada dos puntos de aumento en la adherencia a esta dieta ${ }^{19}$. Además, una evaluación de corte transversal demostró que los pacientes diabéticos tipo 2 con mayor adherencia a DMed tenían menores niveles de hemoglobina glicosilada (Hbalc) y glicemia postprandial ${ }^{20}$. Adicionalmente, en un grupo de pacientes diabéticos que reportaban un mayor índice de DMed, la mortalidad por cualquier causa fue menor en $37 \%$. Dentro de los componentes de esta dieta, los alimentos que más se asociaban a este efecto protector fueron la ingesta moderada de alcohol, el elevado consumo de cereales y verduras y la baja ingesta de lácteos y carnes ${ }^{21}$.

Por último, varios alimentos que forman parte de una DMed han demostrado beneficios en el control de los mecanismos fisiopatológicos involucrados en el hígado graso y la esteatohepatitis no alcohólica. Aunque todavía faltan estudios de mayor calidad metodológica, algunos trabajos pilotos de intervención sugieren que la DMed atenúa la progresión del hígado graso no alcohólico, aunque no contribuye a la prevención de esta patología en pacientes en riesgo ${ }^{22}$.

\section{Dieta mediterránea y cáncer}

Varios estudios observacionales y cohortes sugieren un rol protector de la DMed sobre la incidencia y la mortalidad por cáncer $(\mathrm{Ca})$. En la cohorte EPIC, se observó que la adherencia a la DMed se correlacionaba con una reducción de $24 \%$ en la mortalidad por cáncer durante el seguimiento de cerca de 22.000 individuos por más de 4 años ${ }^{11}$. Asimismo, la subcohorte griega de este mismo estudio demostró una relación inversa entre adherencia a DMed e incidencia general de cáncer (excluyendo Ca cutáneo no melanocítico ${ }^{23}$. Confirmando lo anterior, un meta-análisis basado en la información acumulada de 8 cohortes ( 514.800 sujetos) evidenció que una mayor adherencia a la DMed se asociaba menor incidencia y mortalidad por cáncer ${ }^{24}$.
El menor riesgo general de cáncer en los países mediterráneos se debe principalmente a una menor incidencia de Ca de colon, endometrio, mama y próstata. De hecho, se ha planteado que la DMed podría prevenir 25\% de los casos de Ca colorrectal, 10 a $15 \%$ de Ca prostático y endometrial y 15 a $20 \%$ de Ca mamario ${ }^{25}$. Algunos de los factores dietarios de la DMed que se postulan como responsables de esta protección son el alto consumo de frutas, verduras y cereales integrales, la baja ingesta de carnes rojas, carbohidratos refinados y grasas saturadas junto con el elevado consumo de aceite de oliva ${ }^{26}$. Es así como, a través del adecuado aporte de ácidos grasos omega6/omega3, ácidos grasos monoinsturados, fibra, polifenoles y otros antioxidantes, este patrón dietario ejercería un efecto beneficioso contra el desarrollo de diversos tipos de cánceres ${ }^{27}$. Asimismo el aceite de oliva ha mostrado ser un factor independiente de protección contra varios tipos de cáncer, en especial del aparato respiratorio ${ }^{27}$.

\section{Dieta mediterránea y enfermedades neurodegenerativas}

Las enfermedades neurodegenerativas se caracterizan por una disfunción neuronal progresiva asociada a atrofia de estructuras del sistema nervioso central. Su prevalencia aumenta exponencialmente con la edad, alcanzando $30 \%$ en mayores de 90 años. Dado el envejecimiento de la población, cada año se diagnostican 4,6 millones de nuevos casos de demencia ${ }^{28}$, un importante problema de salud pública y causa de fragilidad y dependencia en la población adulta mayor. Como aún no existe un tratamiento efectivo, las estrategias preventivas para estas condiciones patológicas adquieren cada vez mayor relevancia y la dieta, principalmente la DMed, ha captado un interés progresivo.

Este patrón dietario ha demostrado, en varios estudios epidemiológicos y observacionales, disminuir la incidencia de enfermedades como Alzheimer (EA) y Parkinson, así como un rol beneficioso frente al deterioro cognitivo leve (DCL) asociado al envejecimiento ${ }^{28}$. Algunos de estos trabajos derivan de población no mediterránea que participa en la cohorte WHICAP (Washington Heights-Inwood Columbia Aging Project). Esta cohorte, con un seguimiento de 2.258 individuos durante un período de 4 años, 
mostró que una mayor adherencia a la DMed se asociaba a casi $10 \%$ menor riesgo de $\mathrm{EA}(\mathrm{RR}=$ $0,91)$ y que los individuos en el tercil de mayor adherencia a DMed mostraban $40 \%$ menos riesgo de EA $^{29}$ y $28 \%$ menor riesgo de desarrollar DCL ${ }^{30}$ comparado con los del tercil más bajo. Asimismo, dentro de los individuos que desarrollaron DCL, una mayor adherencia a DMed confería $48 \%$ de menor riesgo de progresión a EA comparado con los casos del tercil inferior. Posteriormente, un estudio caso-control desarrollado en esta misma cohorte demostró que la DMed prevendría la EA en forma independiente de su beneficio sobre patologías asociadas a daño vascular ${ }^{31}$. De hecho, se postula que el efecto antiinflamatorio y antioxidante proveniente de la combinación de alimentos recomendados por la DMed sería, al menos en parte, el responsable de los beneficios observados en patologías neurodegenerativas ${ }^{32}$.

Todos estos hallazgos han sido posteriormente confirmados en dos meta-análisis. El primero -basado en 5 cohortes con un total de 133.626 sujetos- estableció que un aumento de dos puntos en adherencia a DMed se asociaba a $13 \%$ de reducción de riesgo de sufrir alguna enfermedad neurodegenerativa ${ }^{24}$. El segundo estudio obtuvo resultados similares, confirmando el rol beneficioso de la DMed en la prevención de estas enfermedades ${ }^{10}$.

\section{Dieta mediterránea y mortalidad}

Uno de los primeros estudios en mostrar una asociación inversa entre DMed y mortalidad fue el trabajo de Trichopolou y cols. publicado en 1995. Usando un cuestionario semicuantitativo validado de 8 ítems para medir la adherencia a DMed en adultos mayores, en este estudio se estableció que por cada punto de aumento en la adherencia a esta dieta, la mortalidad por cualquier causa disminuía en $17 \%{ }^{3}$. Posteriormente, el mismo equipo mostró, en el seguimiento por 4 años de 22.043 adultos sanos de la cohorte griega del estudio EPIC, que un aumento de 2 puntos en la escala de adherencia a DMed se asociaba a $25 \%$ de reducción en mortalidad general. En este estudio, la reducción de la mortalidad por enfermedad coronaria y por cáncer, fue de $33 \%$ y $24 \%$, respectivamente ${ }^{11}$. Esta asociación detectada en la población griega fue corroborada en un análisis ampliado de las 9 cohortes europeas del estudio EPIC, incluyendo, aproximadamente 74.000 individuos $^{33}$.

\section{Estudios de intervención}

Actualmente existen más de 50 estudios de intervención con DMed midiendo su efecto sobre el peso corporal, parámetros metabólicos glicémicos y lipídicos, inflamación, SM, diabetes y $\mathrm{ECV}^{34}$. La Tabla 1 resume los trabajos clínicos que han involucrado de cientos a miles de pacientes con un período de intervención de al menos 12 meses y usando un grupo control. A continuación revisaremos algunos de los estudios más destacados para diferentes tipos de objetivos clínicos.

\section{El estudio de Lyon y la iniciativa PREDIMED}

El primer trabajo prospectivo de intervención con DMed que evaluó desenlaces clínicos fue el Lyon Heart Study en que 605 individuos con antecedente de infarto al miocardio fueron distribuidos al azar en un grupo manejado con DMed enriquecida con ácido graso alfa-linolénico versus un grupo con dieta control. Al cabo de 27 meses de seguimiento, el consumo de DMed disminuyó la incidencia de eventos coronarios así como la mortalidad coronaria en 73 y $70 \%$, respectivamente. De esta manera, se concluyó que la DMed era una estrategia no farmacológica efectiva a mediano plazo para disminuir los eventos clínicos coronarios en prevención secundaria ${ }^{35}$.

El siguiente estudio de intervención y de gran impacto con respecto a los efectos de la DMed en salud humana ha sido el proyecto PREDIMED (Prevención con Dieta Mediterránea), cuyo objetivo principal fue evaluar los efectos a largo plazo de la DMed en la incidencia de ECV. Este estudio multicéntrico fue realizado en España entre los años 2003 y 2011 e incluyó a 7.447 hombres y mujeres mayores de 50 años de elevado riesgo cardiovascular, pero sin antecedentes de eventos cardiovasculares previos. Los participantes fueron distribuidos al azar en tres grupos: DMed suplementada con aceite de oliva (1 litro/semana), DMed suplementada con frutos secos ( $30 \mathrm{~g} /$ día $)$ o dieta baja en grasas (grupo control), sin restricción del aporte calórico ni promoción de actividad física ${ }^{36}$. Al cabo de 4,8 años de seguimiento, los participantes que consumieron DMed (suplementada con aceite de oliva o frutos secos) presentaron una reducción de $30 \%$ en el riesgo de eventos cardiovasculares, principalmente accidentes cerebrovasculares, comparados con el grupo control ${ }^{37}$, con un efecto 
Tabla 1. Estudios de intervención con dieta mediterránea que incluyen grupo control, tamaño muestral superior a 100 sujetos y tiempo de seguimiento igual o superior a 12 meses

\begin{tabular}{|c|c|c|c|c|c|}
\hline $\begin{array}{l}\text { Autor/año } \\
\text { publicación/ } \\
\text { país }\end{array}$ & $\begin{array}{l}\text { Tipo de } \\
\text { estudio }\end{array}$ & Muestra & $\begin{array}{l}\text { Seguimiento } \\
\text { promedio }\end{array}$ & Outcome & Resultados \\
\hline $\begin{array}{l}\text { De Lorgeril, } \\
\text { 199435; } \\
\text { Francia }\end{array}$ & $\begin{array}{l}\text { Prevención } \\
\text { secundaria }\end{array}$ & $\begin{array}{l}605 \text { individuos con } \\
\text { antecedente de } \\
\text { IAM }\end{array}$ & 27 meses & $\begin{array}{l}\text { Incidencia y } \\
\text { mortalidad por } \\
\text { IAM }\end{array}$ & $\begin{array}{l}73 \% \text { de reducción en eventos } \\
\text { coronarios, } 70 \% \text { de reducción en } \\
\text { mortalidad }\end{array}$ \\
\hline $\begin{array}{l}\text { Bemelmans, } \\
2000^{51} ; \\
\text { Holanda }\end{array}$ & $\begin{array}{l}\text { Prevención } \\
\text { primaria }\end{array}$ & $\begin{array}{l}266 \text { individuos con } \\
\text { hipercolesterolemia } \\
y \geq 2 \text { factores de } \\
\text { RCV }\end{array}$ & 12 meses & $\begin{array}{l}\text { Colesterol } \\
\text { plasmático total } \\
\text { e IMC }\end{array}$ & $\begin{array}{l}2-4 \% \text { de reducción de colesterol } \\
\text { total en mujeres, sin efecto signifi- } \\
\text { cativo en peso corporal }\end{array}$ \\
\hline $\begin{array}{l}\text { Barzi, } \\
2003^{52} \\
\text { Italia }\end{array}$ & $\begin{array}{l}\text { Prevención } \\
\text { secundaria }\end{array}$ & $\begin{array}{l}11.323 \text { individuos } \\
\text { con antecedente } \\
\text { de IAM }\end{array}$ & 6,5 años & $\begin{array}{l}\text { Mortalidad } \\
\text { global }\end{array}$ & $\begin{array}{l}49 \% \text { de reducción en riesgo de } \\
\text { mortalidad en cuartil superior } \\
\text { versus inferior para score de DMed }\end{array}$ \\
\hline $\begin{array}{l}\text { Esposito, } \\
2004^{53} \\
\text { Italia }\end{array}$ & $\begin{array}{l}\text { Prevención } \\
\text { primaria }\end{array}$ & 180 individuos & 2 años & $\begin{array}{l}\text { Función endote- } \\
\text { lial, marcadores } \\
\text { de inflamación } \\
\text { y SM }\end{array}$ & $\begin{array}{l}\text { Mejoría de parámetros metabó- } \\
\text { licos, inflamatorios y fisiológicos } \\
\text { junto con } 51 \% \text { de reducción en } \\
\text { prevalencia de SM }\end{array}$ \\
\hline $\begin{array}{l}\text { Salas Salvado, } \\
2011^{38} ; \\
\text { España }\end{array}$ & $\begin{array}{l}\text { Prevención } \\
\text { primaria }\end{array}$ & $\begin{array}{l}418 \text { individuos no } \\
\text { diabéticos }\end{array}$ & 4 años & $\begin{array}{l}\text { Incidencia de } \\
\text { DM2 }\end{array}$ & $\begin{array}{l}52 \% \text { de reducción en la incidencia } \\
\text { de } \mathrm{DM} 2\end{array}$ \\
\hline $\begin{array}{l}\text { Estruch, } \\
2013^{37} ; \\
\text { España }\end{array}$ & $\begin{array}{l}\text { Prevención } \\
\text { primaria }\end{array}$ & $\begin{array}{l}7.447 \text { individuos } \\
\text { con RCV elevado }\end{array}$ & 4,8 años & $\begin{array}{l}\text { Eventos y mor- } \\
\text { talidad por ECV }\end{array}$ & $\begin{array}{l}30 \% \text { de reducción en eventos } \\
\text { cardiovasculares }\end{array}$ \\
\hline $\begin{array}{l}\text { Babio, } \\
2014^{41} \\
\text { España }\end{array}$ & $\begin{array}{l}\text { Prevención } \\
\text { primaria y } \\
\text { seundaria }\end{array}$ & $\begin{array}{l}1.919 \text { participantes } \\
\text { sin SM y } 3.392 \\
\text { sujetos con SM }\end{array}$ & 4,8 años & $\begin{array}{l}\text { Incidencia y } \\
\text { reversión de SM }\end{array}$ & $\begin{array}{l}28-35 \% \text { de mayor tasa de rever- } \\
\text { sión en SM, sin efecto sobre la } \\
\text { incidencia de SM }\end{array}$ \\
\hline $\begin{array}{l}\text { Valls-Pedret, } \\
2015^{42} \\
\text { España }\end{array}$ & $\begin{array}{l}\text { Prevención } \\
\text { primaria }\end{array}$ & 447 sujetos & 4,1 años & $\begin{array}{l}\text { Cambios cogni- } \\
\text { tivos }\end{array}$ & $\begin{array}{l}\text { Mejoría significativa en puntajes } \\
\text { de tests cognitivos en sujetos } \\
\text { sometidos a DMed suplementada } \\
\text { con aceite de oliva }\end{array}$ \\
\hline $\begin{array}{l}\text { Toledo, } \\
\text { 201543; } \\
\text { España }\end{array}$ & $\begin{array}{l}\text { Prevención } \\
\text { primaria }\end{array}$ & 4.152 sujetos & 4,8 años & $\begin{array}{l}\text { Incidencia de } \\
\text { cáncer mamario }\end{array}$ & $\begin{array}{l}68 \% \text { menor incidencia de cáncer } \\
\text { mamario en mujeres sometidas a } \\
\text { DMed suplementada con aceite } \\
\text { de oliva }\end{array}$ \\
\hline
\end{tabular}

IAM: infarto agudo al miocardio; RCV: Riesgo cardiovascular; IMC: índice de masa corporal; DMed: dieta mediterránea; SM: síndrome metabólico; DM2: diabetes mellitus tipo 2; ECV: enfermedad cardiovascular.

protector de magnitud comparable a aquél otorgado por medidas farmacológicas como el uso de estatinas. Por otro lado, análisis adicionales del mismo estudio reportaron que la incidencia de diabetes fue significativamente menor entre los sujetos no diabéticos de los grupos de DMed, con una reducción de $52 \%$ en la aparición de nuevos casos de diabetes comparado con el grupo $\mathrm{control}^{38}$. Adicionalmente, un análisis post-hoc reciente de este estudio mostró un beneficio de este patrón dietario en la prevención de retinopatía diabética ${ }^{39}$. Asimismo, ambas DMed mostraron beneficios sobre diferentes factores de riesgo CV como dislipidemia, resistencia a la insulina, inflamación, oxidación y ateroesclerosis carotídea ${ }^{36}$. Además, la DMed tuvo un efecto favorable sobre el SM, mejorando sus componentes y disminuyendo su prevalencia por medio de un aumento en la reversión de esta condición con respecto al estado basal ${ }^{40,41}$. Finalmente, este mismo estudio PREDIMED demostró recientemente que esta dieta podría disminuir el deterioro cognitivo aso- 


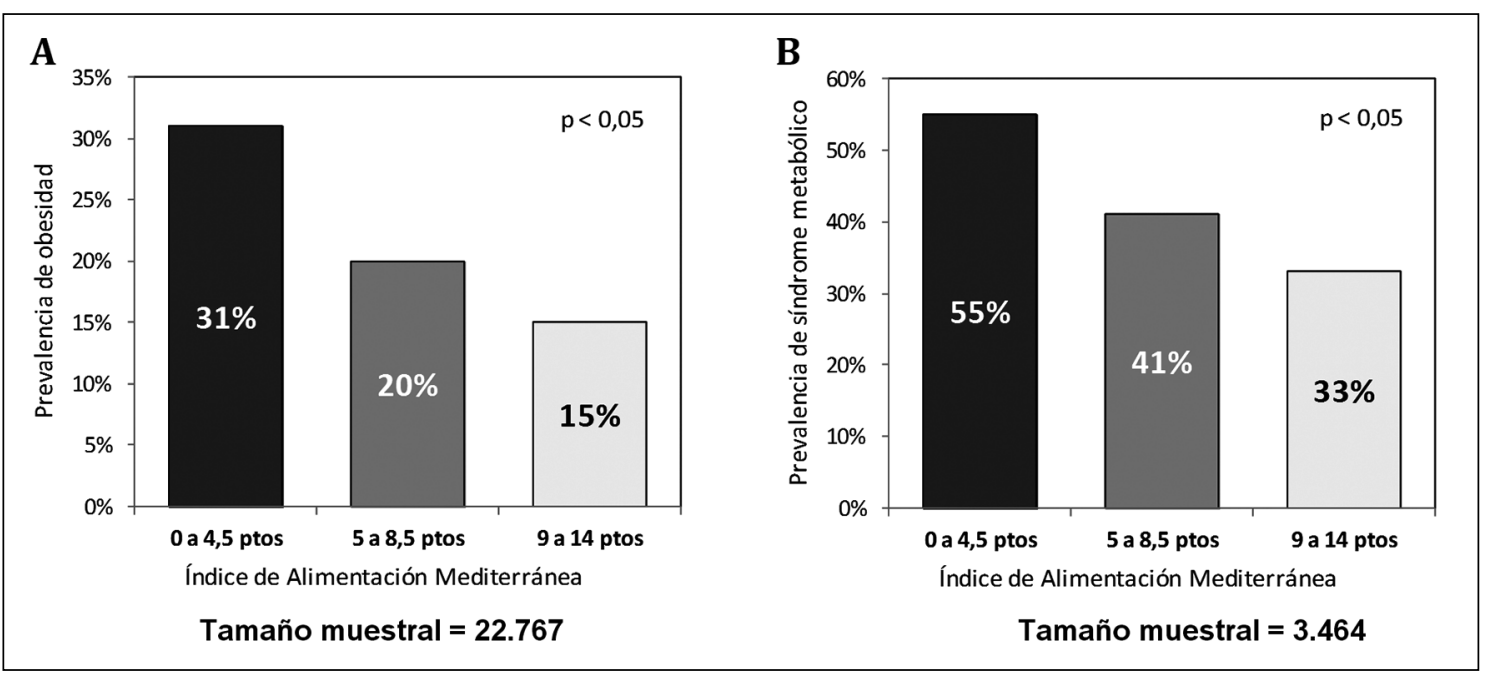

Figura 2. Asociación entre índice de alimentación mediterránea (IAM) y prevalencia de obesidad (A) y síndrome metabólico (B) en población adulta chilena registrada en el Programa Aliméntate Sano de la Pontificia Universidad Católica de Chile.

ciado al envejecimiento ${ }^{42}$, así como la incidencia de cáncer de mama ${ }^{43}$.

Todos estos beneficios asociados al consumo de una DMed fueron independientes de cambios en el peso corporal, lo que sugiere un efecto intrínseco -más allá de un ajuste del balance energético- de este patrón dietario ${ }^{44}$. De esta manera, el estudio PREDIMED ha aportado evidencia científica contundente con respecto al rol beneficioso de la DMed en la prevención de ECV y de otras enfermedades crónicas, confirmando lo previamente establecido en el estudio de Lyon, y validando la evidencia derivada de los múltiples estudios observacionales transversales y longitudinales realizados desde la década de 1960.

\section{Aplicando la dieta mediterránea en Chile: estudios de asociación e intervención}

La zona central de nuestro país constituye uno de los cinco ecosistemas mediterráneos del mundo, donde naturalmente se producen los alimentos característicos de la DMed. Por lo tanto, promover el consumo de este tipo de productos nacionales ofrece una gran oportunidad para el manejo de la creciente incidencia de enfermedades crónicas en nuestro país ${ }^{44}$. De hecho, datos del Programa Aliméntate Sano de nuestra institución indican que los adultos chilenos que exhiben un patrón de alimentación más mediterráneo (puntaje $\geq 9$ puntos en un índice de alimentación mediterránea de 0 a 14 puntos), presentan una menor prevalencia de obesidad y SM (Figura 2).

Por otro lado, algunos estudios pilotos de intervención con DMed han reproducido sus beneficios en población de nuestro país. En el primero de ellos, se evaluó la factibilidad de una mediterranización de la dieta durante un año en un grupo de trabajadores dentro del contexto laboral y su efecto sobre la prevalencia de SM y sus componentes. La intervención consistió en una adecuación mediterránea de los alimentos ofrecidos en el casino del lugar de trabajo (aumento del consumo de verduras, frutas, pescado, pan integral y aceite de oliva y disminución de la ingesta de carnes rojas) junto con charlas educativas sobre DMed y salud, aconsejando opciones de alimentos saludables para las comidas extralaborales. Durante esta intervención, se observó una efectiva mediterranización de la dieta de los participantes, medida por medio de un índice de DMed, y una mejoría significativa de algunos componentes (obesidad abdominal, hipertensión arterial y colesterol HDL bajo) del SM. De hecho, al cabo de los 12 meses se observó 48\% de reversión de los casos iniciales de $\mathrm{SM}$, con una disminución en la prevalencia total de esta condición clínica desde $24 \%$ al inicio del estudio a $16 \%$ al término de éste ${ }^{45}$. 
En el segundo estudio se evaluó el posible efecto protector de la DMed -con o sin el consumo concomitante de vino tinto- sobre el estrés oxidativo, comparándolo con una dieta típica occidental en un grupo de adultos sanos durante un período de 3 meses. Al cabo de este tiempo se observó que los voluntarios asignados a la DMed mostraban mayor capacidad antioxidante y menor daño oxidativo $^{46}$, mejor perfil de ácidos grasos ${ }^{47} \mathrm{y}$ mejores parámetros hemostáticos plasmáticos ${ }^{48}$, junto con una mejor función endotelial ${ }^{49}$, que aquellos alimentados con dieta occidental. Además, el consumo moderado de vino tinto mejoró la capacidad antioxidante en ambos grupos, siendo capaz de contrarrestar el daño oxidativo observado por la ingesta de la dieta occidental. Finalmente, un estudio reciente ha reportado que el consumo de una DMed hipocalórica en mujeres premenopáusicas con sobrepeso u obesidad redujo los niveles séricos de carboximetilcisteína, un indicador de la presencia de productos de glicación avanzada ${ }^{50}$.

\section{Conclusiones}

El estilo de alimentación mediterráneo ha demostrado, con amplia solvencia científica, por asociación o intervención, generar numerosos beneficios en la prevención y tratamiento de diferentes tipos de condiciones de riesgo y/o patologías crónicas. Nuestro país posee uno de los cinco ecosistemas mediterráneos del mundo, siendo su producción agrícola y acuícola local muy abundante en productos asociados a la DMed. Además, existen estudios que indican que los beneficios de la DMed son replicables en países fuera de la cuenca mediterránea, incluyendo Chile. En conjunto, toda la evidencia revisada sugiere que la alimentación de tipo mediterráneo es una importante herramienta para ser implementada a nivel de salud pública en el desarrollo de políticas efectivas para disminuir la morbimortalidad prematura en la población chilena.

\section{Dedicatoria}

Este artículo está dedicado a la memoria del Dr. Federico Leighton Puga (1938-2012), médico investigador y pionero en el estudio y la promoción de la dieta mediterránea en Chile.
Agradecimientos: Los resultados de nuestro grupo mencionados en este artículo han sido obtenidos mediante proyectos financiados por Fundación Alimenta y Fundación Banmédica.

\section{Referencias}

1. Mozaffarian D, Appel LJ, Van Horn L. Components of a cardioprotective diet: new insights. Circulation 2011; 123 (24): 2870-91.

2. Sofi F, Macchi C, Abbate R, Gensini GF, Casini A. Mediterranean diet and health. Biofactors 2013; 39 (4): 335-42.

3. Trichopoulou A, Kouris-Blazos A, Wahlqvist ML, Gnardellis C, Lagiou P, Polychronopoulos E, et al. Diet and overall survival in elderly people. BMJ 1995; 311 (7018): 1457-60.

4. Alberti-Fidanza A, Fidanza F. Mediterranean Adequacy Index of Italian diets. Public Health Nutr 2004; 7 (7): 937-41.

5. Martínez-González MA, Fernández-Jarne E, Serrano-Martínez M, Wright M, Gómez-Gracia E. Development of a short dietary intake questionnaire for the quantitative estimation of adherence to a cardioprotective Mediterranean diet. Eur J Clin Nutr 2004; 58 (11): 1550-2.

6. Fung TT, Hu FB, McCullough ML, Newby PK, Willett WC, Holmes MD. Diet quality is associated with the risk of estrogen receptor-negative breast cancer in postmenopausal women. J Nutr 2006; 136 (2): 466-72.

7. Trichopoulou A, Bamia C, Trichopoulos D. Anatomy of health effects of Mediterranean diet: Greek EPIC prospective cohort study. BMJ 2009; 338: b2337.

8. Buckland G, Agudo A, Travier N, Huerta JM, Cirera L, Tormo MJ, et al. Adherence to the Mediterranean diet reduces mortality in the Spanish cohort of the European Prospective Investigation into Cancer and Nutrition (EPIC-Spain). Br J Nutr 2011; 106 (10): 1581-91.

9. Knoops KT, de Groot LC, Kromhout D, Perrin AE, Moreiras-Varela O, Menotti A, et al. Mediterranean diet, lifestyle factors, and 10-year mortality in elderly European men and women: the HALE project. JAMA 2004; 292 (12): 1433-9.

10. Sofi F, Abbate R, Gensini GF, Casini A. Accruing evidence on benefits of adherence to the Mediterranean diet on health: an updated systematic review and meta-analysis. Am J Clin Nutr 2010; 92 (5): 1189-96.

11. Trichopoulou A, Costacou T, Bamia C, Trichopoulos D. Adherence to a Mediterranean diet and survival in a Greek population. N Engl J Med 2003; 348 (26): 2599608. 
12. Trichopoulou A, Bamia C, Norat T, Overvad K, Schmidt EB, Tjonneland A, et al. Modified Mediterranean diet and survival after myocardial infarction: the EPIC-Elderly study. Eur J Epidemiol 2007; 22 (12): 871-81.

13. Martínez-González MA, García-López M, Bes-Rastrollo M, Toledo E, Martínez-Lapiscina EH, Delgado-Rodríguez $\mathrm{M}$, et al. Mediterranean diet and the incidence of cardiovascular disease: a Spanish cohort. Nutr Metab Cardiovasc Dis 2011; 21 (4): 237-44.

14. Buckland G, González CA, Agudo A, Vilardell M, Berenguer A, Amiano P, et al. Adherence to the Mediterranean diet and risk of coronary heart disease in the Spanish EPIC Cohort Study. Am J Epidemiol 2009; 170 (12): 1518-29.

15. Mitrou PN, Kipnis V, Thiebaut AC, Reedy J, Subar AF, Wirfalt E, et al. Mediterranean dietary pattern and prediction of all-cause mortality in a US population: results from the NIH-AARP Diet and Health Study. Arch Intern Med 2007; 167 (22): 2461-8.

16. Sofi F, Macchi C, Abbate R, Gensini GF, Casini A. Mediterranean diet and health status: an updated meta-analysis and a proposal for a literature-based adherence score. Public Health Nutr 2014; 17 (12): 2769-82.

17. Kastorini CM, Milionis HJ, Esposito K, Giugliano D, Goudevenos JA, Panagiotakos DB. The effect of Mediterranean diet on metabolic syndrome and its components: a meta-analysis of 50 studies and 534,906 individuals. J Am Coll Cardiol 2011; 57 (11): 1299-313.

18. Nordmann AJ, Suter-Zimmermann K, Bucher HC, Shai I, Tuttle KR, Estruch R, et al. Meta-analysis comparing Mediterranean to low-fat diets for modification of cardiovascular risk factors. Am J Med 2011; 124 (9): 841-51 e2.

19. Martínez-González MA, de la Fuente-Arrillaga C, Nunez-Córdoba JM, Basterra-Gortari FJ, Beunza JJ, Vazquez Z, et al. Adherence to Mediterranean diet and risk of developing diabetes: prospective cohort study. BMJ 2008; 336 (7657): 1348-51.

20. Esposito K, Maiorino MI, Di Palo C, Giugliano D, Campanian Postprandial Hyperglycemia Study G. Adherence to a Mediterranean diet and glycaemic control in Type 2 diabetes mellitus. Diabet Med 2009; 26 (9): 900-7.

21. Bonaccio M, Di Castelnuovo A, Costanzo S, Persichillo M, De Curtis A, Donati MB, et al. Adherence to the traditional Mediterranean diet and mortality in subjects with diabetes. Prospective results from the MOLI-SANI study. Eur J Prev Cardiol 2015.

22. Velasco N, Contreras A, Grassi B. The Mediterranean diet, hepatic steatosis and nonalcoholic fatty liver disease. Curr Opin Clin Nutr Metab Care 2014; 17 (5): 453-7.
23. Benetou V, Trichopoulou A, Orfanos P, Naska A, Lagiou $\mathrm{P}$, Boffetta $\mathrm{P}$, et al. Conformity to traditional Mediterranean diet and cancer incidence: the Greek EPIC cohort. Br J Cancer 2008; 99 (1): 191-5.

24. Sofi F, Cesari F, Abbate R, Gensini GF, Casini A. Adherence to Mediterranean diet and health status: meta-analysis. BMJ 2008; 337: a1344.

25. Trichopoulou A, Lagiou P, Kuper H, Trichopoulos D. Cancer and Mediterranean dietary traditions. Cancer Epidemiol Biomarkers Prev 2000; 9 (9): 869-73.

26. La Vecchia C. Mediterranean diet and cancer. Public Health Nutr 2004; 7 (7): 965-8.

27. Giacosa A, Barale R, Bavaresco L, Gatenby P, Gerbi V, Janssens J, et al. Cancer prevention in Europe: the Mediterranean diet as a protective choice. Eur J Cancer Prev 2013; 22 (1): 90-5.

28. Sofi F, Macchi C, Casini A. Mediterranean Diet and Minimizing Neurodegeneration. Curr Nutr Rep 2013; 2: $75-80$.

29. Scarmeas N, Stern Y, Tang MX, Mayeux R, Luchsinger JA. Mediterranean diet and risk for Alzheimer's disease. Ann Neurol 2006; 59 (6): 912-21.

30. Scarmeas N, Stern Y, Mayeux R, Manly JJ, Schupf N, Luchsinger JA. Mediterranean diet and mild cognitive impairment. Arch Neurol 2009; 66 (2): 216-25.

31. Scarmeas N, Stern Y, Mayeux R, Luchsinger JA. Mediterranean diet, Alzheimer disease, and vascular mediation. Arch Neurol 2006; 63 (12): 1709-17.

32. Feart C, Samieri C, Barberger-Gateau P. Mediterranean diet and cognitive function in older adults. Curr Opin Clin Nutr Metab Care 2010; 13 (1): 14-8.

33. Trichopoulou A, Orfanos P, Norat T, Bueno-de-Mesquita B, Ocke MC, Peeters PH, et al. Modified Mediterranean diet and survival: EPIC-elderly prospective cohort study. BMJ 2005; 330 (7498): 991.

34. Serra-Majem L, Roman B, Estruch R. Scientific evidence of interventions using the Mediterranean diet: a systematic review. Nutr Rev 2006; 64 (2 Pt 2): S27-47.

35. de Lorgeril M, Renaud S, Mamelle N, Salen P, Martin JL, Monjaud I, et al. Mediterranean alpha-linolenic acid-rich diet in secondary prevention of coronary heart disease. Lancet 1994; 343 (8911): 1454-9.

36. Martínez-González MA, Salas-Salvado J, Estruch R, Corella DD, Fito M, Ros E, et al. Benefits of the Mediterranean Diet: Insights from the PREDIMED Study. Prog Cardiovasc Dis 2015.

37. Estruch R, Ros E, Salas-Salvado J, Covas MI, Corella $\mathrm{D}$, Aros F, et al. Primary prevention of cardiovascular disease with a Mediterranean diet. N Engl J Med 2013; 368 (14): 1279-90.

38. Salas-Salvado J, Bullo M, Babio N, Martínez-González 
MA, Ibarrola-Jurado N, Basora J, et al. Reduction in the incidence of type 2 diabetes with the Mediterranean diet: results of the PREDIMED-Reus nutrition intervention randomized trial. Diabetes Care 2011; 34 (1): 14-9.

39. Díaz-López A, Babio N, Martínez-González MA, Corella D, Amor AJ, Fito M, et al. Mediterranean Diet, Retinopathy, Nephropathy, and Microvascular Diabetes Complications: A Post Hoc Analysis of a Randomized Trial. Diabetes Care 2015.

40. Salas-Salvado J, Fernández-Ballart J, Ros E, Martínez-González MA, Fito M, Estruch R, et al. Effect of a Mediterranean diet supplemented with nuts on metabolic syndrome status: one-year results of the PREDIMED randomized trial. Arch Intern Med 2008; 168 (22): 2449-58.

41. Babio N, Toledo E, Estruch R, Ros E, Martínez-González MA, Castaner O, et al. Mediterranean diets and metabolic syndrome status in the PREDIMED randomized trial. CMAJ 2014; 186 (17): E649-57.

42. Valls-Pedret C, Sala-Vila A, Serra-Mir M, Corella D, de la Torre R, Martínez-González MA, et al. Mediterranean Diet and Age-Related Cognitive Decline: A Randomized Clinical Trial. JAMA Intern Med 2015.

43. Toledo E, Salas-Salvado J, Donat-Vargas C, Buil-Cosiales P, Estruch R, Ros E, et al. Mediterranean Diet and Invasive Breast Cancer Risk Among Women at High Cardiovascular Risk in the PREDIMED Trial: A Randomized Clinical Trial. JAMA Intern Med 2015: 1-9.

44. Urquiaga I, Echeverría G, Dussaillant C, Rigotti A. Origen, Componentes y Posibles Mecanismos de Acción de la Dieta Mediterránea. Rev Med Chile 2015; Artículo en prensa.

45. Leighton F, Polic G, Strobel P, Pérez D, Martínez C, Vasquez L, et al. Health impact of Mediterranean diets in food at work. Public Health Nutr 2009; 12 (9A): 1635-43.

46. Urquiaga I, Strobel P, Pérez D, Martínez C, Cuevas A, Castillo O, et al. Mediterranean diet and red wine protect against oxidative damage in young volunteers. Atherosclerosis 2010; 211 (2): 694-9.

47. Urquiaga I, Guasch V, Marshall G, San Martin A, Castillo O, Rozowski J, et al. Effect of Mediterranean and Occidental diets, and red wine, on plasma fatty acids in humans. An intervention study. Biol Res 2004; 37 (2): 253-61.

48. Mezzano D, Leighton F, Martínez C, Marshall G, Cuevas A, Castillo O, et al. Complementary effects of Mediterranean diet and moderate red wine intake on haemostatic cardiovascular risk factors. Eur J Clin Nutr 2001; 55 (6): 444-51.

49. Leighton F, Cuevas A, Guasch V, Pérez DD, Strobel P, San Martín A, et al. Plasma polyphenols and antioxidants, oxidative DNA damage and endothelial function in a diet and wine intervention study in humans. Drugs Exp Clin Res 1999; 25 (2-3): 133-41.

50. Rodríguez JM, Leiva Balich L, Concha MJ, Mizon C, Bunout Barnett D, Barrera Acevedo G, et al. Reduction of serum advanced glycation end-products with a low calorie Mediterranean diet. Nutr Hosp 2015; 31 (6): 2511-7. 